\title{
The Next Wave: Confexting
}

\author{
Lubdha M. Shah • Edward P. Quigley • \\ Richard H. Wiggins III
}

Published online: 6 July 2011

(C) Society for Imaging Informatics in Medicine 2011

\begin{abstract}
Radiology conferences enable participants the opportunity to ask experts questions through question and answer ( $\mathrm{Q}$ and $\mathrm{A}$ ) sessions or individually. Given the time limitations and intimidating circumstances, we incorporated conference text messaging (confexting) as a method of increasing interactivity between the audience and speakers. During a 5-day radiology conference, text messaging was utilized for anonymous interactivity between the audience and speakers during Q and A sessions. There were 324 text messages; 76 of these were either follow-up statements or questions related to earlier text messages. Forty-two questions were submitted via paper notes. There was a general trend of an increasing number of text messages and a decreasing number of paper notes. The anonymous text messaging system was found to be an effective method for interactivity between the audience and the speakers. The questions and answers could be presented in a PowerPoint format at the formal Q and A sessions. Questions texted to the authors during their talks could be immediately
\end{abstract}

\section{M. Shah}

Departments of Radiology and Neurosurgery,

University of Utah Health Sciences Center,

30 North, 1900 East, \#1A71,

Salt Lake City, UT 84132-2140, USA

E. P. Quigley

Department of Radiology,

University of Utah Health Sciences Center,

30 North, 1900 East, \#1A71,

Salt Lake City, UT, USA

R. H. Wiggins III $(\square)$

Departments of Radiology, Otolaryngology and Head and Neck

Surgery, and BioMedical Informatics,

University of Utah Health Sciences Center,

30 North, 1900 East, \#1A71,

Salt Lake City, UT 84132-2140, USA

e-mail: Richard.Wiggins@hsc.utah.edu answered or addressed in subsequent talks. Although difficult for some individuals to embrace technology, confexting allows for interactivity and prompts discussion. Confexting is an effective method for interactivity between the audience and speakers not previously utilized in a conference setting. The anonymity and asynchronous communication enable conference participants to submit more questions than in the traditional setting. The speakers may be able to explain more thoroughly difficult concepts more thoroughly with additional slides at $\mathrm{Q}$ and $\mathrm{A}$ sessions or may immediately answer texted questions during their talks.

Keywords Teaching · Continuing medical education . Computer hardware Communication · Education .

Medical · Experiential $\cdot$ Imaging informatics $\cdot$ User interface

\section{Introduction}

As we find more and more technology surrounding us, we want to be certain that we are using it effectively, especially with radiology education. There is a general tendency to overuse new technologies, which can lead to confusion, rather than as an assisting device when attempting to teach a complicated subject. Over the past decade, a variety of technology-driven learning strategies, variously referred to as computer-based, distributed, Web-based, Internet-based, and/or online learning, have been described for use in medical education [1-5]. In a study evaluating radiology education, $97 \%$ of surveyed radiologists stated that they used the internet for radiology education [6]. Though $45 \%$ of participants in the survey said they used the internet for continuing medical education (CME), an institutionally run course was selected most frequently as the preferred method of CME, as well as the most effective and efficient [6]. In this era of smart phones and mobile interactive 
devices, a majority of us, particularly those of us in medicine, are able to receive instant information and immediate feedback. We have used this wave of technology as a novel educational tool in our conferences.

The Accreditation Council for Continuing Medical Education (ACCME) accredits organizations that grant CME credits to individuals who participate in approved educational activities, including conferences [7]. Accreditation requires detailed description of the objectives of a conference and methods and delivery of the educational material. The mission of educational conferences is to improve the CME learning and interactivity. The ACCME promotes multi-lateral learning methods to better encourage and enhance physicians and other health care professionals' learning experience. The ACCME is phasing in new criteria for accreditation from 2008 to 2012, requiring CME providers to assess the impact of their interventions [8].

The actual content of conferences is varied. Some conferences are an overview of radiology while others are an intensive review of a radiology subspecialty. Conference attendees may include general radiologists, subspecialists, and radiology trainees, as well as non-radiology physicians and technologists. The audiences will have large variations in knowledge and skills, particularly based on their practices. It can be difficult to deliver meaningful education to such a diverse group of participants simultaneously. Conference organizers try to carefully prepare with specific objectives for the event with digital and/or hardcopy material to supplement the didactic and case presentations. However, it is often difficult to gauge the educational level of a diverse audience, and many participants may be left with questions. Since the days of radiology residency onward, most practitioners can recall personal experiences of the "hot seat conference." In the conference setting, an individual may not be put "on the spot" but nevertheless may feel intimidated at asking questions. These questions may be perplexing other learners as well. It would also be helpful for speakers to have an understanding of the comprehension of the audience during and after their talks.

Radiology conferences increasingly enable participants the opportunity to question experts in the field. Traditionally, question and answer (Q and $\mathrm{A})$ sessions have involved speakers at a podium with attendees posing questions in front of the audience. Alternatively, attendees may approach individual speakers after the session, but the time frame is limited and both of these scenarios can be intimidating. Furthermore, it may be difficult for a speaker to accurately convey and quickly describe a complex concept. Audience response systems have been used to allow an indirect interaction with the panel/speaker asking questions to the audience, but not for specific questions from the audience, instead of from the speaker. Given these limitations, we incorporated text messaging as a method of improving interactivity between the speakers and audience and a CME conference.

\section{Materials and Methods}

During a week-long radiology CME conference, text messaging was utilized for anonymous interactivity between the audience and the speakers. A standard cell phone with text messaging capabilities was engaged for the conference. There were approximately 200 (194) conference attendees at the conference, who were a mix in age range, with a slight male predominance. The audience was predominantly from North America, with additional attendees from Europe, the Middle East, Asia, and Australia. The anonymous text messaging concept was explained to the conference attendees at the beginning of the conference. The course director elaborated on the method of interactivity using "confexting" (or conference texting):

"I want to introduce you to Confexting. Years ago, I was always hesitant to ask questions out loud at conferences in front of my peers. I was nervous that I would say something stupid or have a dumb question, and I now know that this hindered my own education. I also know now that if I have a question or don't understand something, there is a very good chance that others in the audience are thinking the exact same thing, and I have been thinking about how to improve our interactivity during this conference.

I have this cell phone during this week, with the number you see on the screens, and published in your syllabus. Please put your phone on silent or vibrate mode, but if you think of any question about anything during this week at any time, 24 hours a day, simply text your question to this number. You know that you will be talking to me, but I have no idea who you are. I will take your question and make it into a PowerPoint slide and I will have the speaker panel address your question at the next Q and A session."

The cell phone number was published in the syllabus for the conference with a Quick Response (QR) code for quick access, and also was displayed periodically during the conference. Questions and answer sessions were scheduled prior to lunch and at the end of the day throughout the course. At these sessions, all of the speakers from the prior morning or afternoon sessions would return to the podium and the course director would moderate the session, presenting the questions and allowing the appropriate speaker to address the question at hand. The speakers would then attempt to answer all questions at the $\mathrm{Q}$ and $\mathrm{A}$ session, often leading to excellent interactive sessions with the speakers sharing their knowledge and experiences with the audience. 


\section{Results}

One of the course directors controlled the conference phone at all times during the conference week. During the conference, there were a total of 671 text messages sent to and from the conference phone. During the conference week, there were 347 outgoing messages and 324 incoming text messages. Of the incoming 324 messages, 76 of these were either follow-up statements or questions related to earlier text messages or general comments to the speakers or the course directors. There were also several text messages sent to the program director while he was giving his didactic talks, which allowed him to immediately address questions from the audience and then continue with his talk. A majority of the outgoing text messages from the conference cell phone were simply confirming with the attendees that their question had been received, or a confirmatory question to be certain that the attendee's questions were answered appropriately. Some attendees opted to submit their questions via paper notes (42).

There was a general trend during the conference of an increasing number text messages and a decreasing number of paper, handwritten questions submitted to the course director (Table 1). The final day of the conference was a half day, ending at noon, explaining the decrease in the number of questions on that day. The reported location of the cell phones of the incoming text messages was similar to the stated origins of the conference attendees, predominantly from North America, from California to Quebec providence, and from Florida to British Columbia. There were a few foreign cellular numbers registered, likely decreased secondary to foreign attendees choosing to acquire regional, more inexpensive prepaid cellular phones during the conference.

\section{Discussion}

There have been numerous technologic advances that allow audience participation through the use of keypads or wireless interfaces, which lend even more of an interactive element to didactic talks [7]. In order to best interact with conference attendees, a social networking type of interaction was utilized. In the past, with a smaller number of attendees, more interactivity was possible with one-on-one interaction between the speakers and the attendees. This conference includes both didactic lectures and interactive case sessions, in which unknown radiology cases are presented to the audience and the diagnostic approach to solving the case is discussed by the speaker and attendee together, but this had become more difficult as the conference audience continued to grow over the past decade. Confexting is now allowing the conference to return to learner-centered teaching from instructor-centered teaching. Rather than focusing on information delivery, confexting allows exploration of the learners' thought processes [8].

The authors found that the anonymous text messaging system was an effective method for interactivity between the audience and the speakers. This method of conference interactivity was found to be extremely popular with both speakers and the audience. Favorable comments were given regarding confexting in the evaluation forms submitted by attendees as required by the ACCME. The texted questions can be answered in a PowerPoint format at the formal Q and A sessions.

This method was also popular with the speakers as they were not equally on the spot in having to answer sometimes complicated questions, and this also allows the speakers to create additional slides to better explain complicated concepts at the Q and A sessions. Some questions can be incorporated or addressed by speakers in subsequent talks. Questions texted to the authors during their talks can be immediately answered, allowing for immediate interactivity and feedback not previously utilized in a conference setting.

A few attendees preferred to write down their questions and hand them in to the course director. Although it may be difficult for some individuals to embrace technology, text messaging enables interactivity, collaboration, and further discussion of issue and new ideas between the audience and the speakers. This is actually a very interesting shift in the form of this conference interactivity from the bland didactic lecture, where the speaker is describing what he/she thinks

Table 1 Chart listing the numbers questions submitted during the conference separated by type for each day of the conference, for the time periods from midnight to midnight

\begin{tabular}{lccccc}
\hline Type of questions submitted & Monday & Tuesday & Wednesday & Thursday & Friday (half day) \\
\hline Digital text (confexting) & 66 & 59 & 77 & 93 & 29 \\
Hard copy paper & 9 & 13 & 6 & 11 & 3 \\
\hline
\end{tabular}

The digital text message (confexting) questions are listed in the middle row, and the hard copy, handwritten paper submitted questions are numbered in the bottom row. Although the numbers are not statistically significant, there is a general trend towards increasing digital questions and decreasing hard copy, handwritten questions during the week, suggesting the general acceptance of the technology by the audience 
is important about a particular subject, to a learner-centered format where the speaker can better determine what is actually important to the audience.

\section{Advantages}

There are several significant advantages of this confexting technique for conference interactivity. One of the interesting outcomes of this method is that the audience was very accepting of the system, beginning to send text message questions immediately at the beginning of the conference, with an increasing use throughout the conference, clearly demonstrating a general acceptance of the method (Fig. 1). There was also the clear appreciation of confexting by the audience as a whole in that the question and answer sessions were conducted without anyone in the audience ever speaking out loud. There were microphones placed around the audience specifically for these sessions during the conference, in case someone in the audience preferred this method, but they were never used. The audience was always eager to hear the question and answer discussions with the speakers. They were willing to stay late for these sessions, even if they continued 20 min late in the day, after a full day from 8 a.m. to 5 p.m. of lectures.

The confexting was also quickly accepted by the speakers themselves, for several reasons. One interesting effect was the control over the question length. The audience was forced to confine their questions into short text strings to quickly get to the important issues without

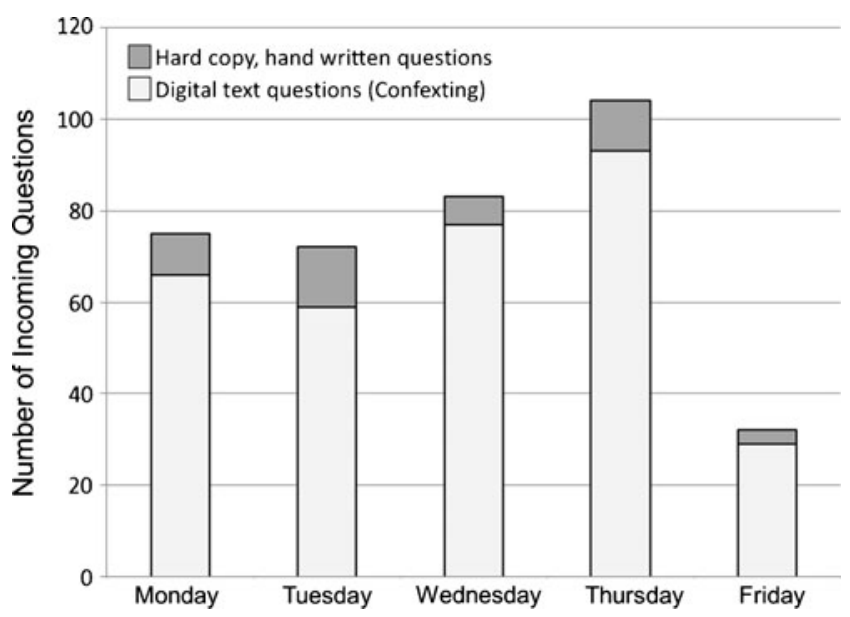

Fig. 1 Graph displaying the total number of hard copy, handwritten questions submitted (dark gray) and the total number of incoming submitted text messages (confexting) (light gray) to the conference cell phone during the conference, with the total height of each bar being the combined number of incoming analog and digital questions together, and showing the general trend of increasing text messages during the week and decreasing paper submitted questions during the week, demonstrating a general acceptance of confexting superfluous background information. There were no text messages that included several minutes of extraneous setup, such as are often seen at live conferences: "Hi. My name is George, and I appreciated your talk, thank you for that. I work in a large group of 40 radiologists, and we live in a small town east of the Mississippi, and I am 5 years out of my training, but I thought once years ago when I was at a conference that I thought I heard someone talk about a technique that might have been used for something like what you were talking about earlier...." This was greatly appreciated and commented on by both the speakers and the audience as a whole.

The confexting method also allowed the speakers time not only to contemplate the question and formulate an answer but also to assemble slides when appropriate to better answer the questions. When the speaker finished a specific talk, he/she was shown the text questions while other talks were finishing so that he/she could formulate an answer for the next question and answer session.

\section{Logistics}

The confexting technique does require a cellular device which allows easy texting capabilities. It also assumes that mobile connectivity is available in the conference venue. The confexting method was attempted at an earlier conference; however, the particular conference room being used had little to no cellular connectivity, making this technique unusable. There were also a few dollars of additional charges for text messages to and from foreign cellular phone numbers. Although an internet connection may not be readily available for all participants at a conference, cellular phone technology is now usually ubiquitous. Confexting seems a natural incorporation of the immediate information retrieval to which we are all growing accustomed. As smart phones evolve, we now find ourselves with small computers in our pockets, like a mini laptop, which may be used continuously throughout the day, but only rarely for voice communication.

\section{Conclusion}

Confexting, or conference texting, is an effective method for improved interactivity between conference audience and speakers. The anonymity and laxity of time enable conference participants to submit more questions than in the traditional setting. The speakers may be able to more thoroughly explain difficult concepts with additional slides at $\mathrm{Q}$ and A sessions, may address questions in subsequent talks, or may immediately answer questions from the audience during their talks. Confexting allows for enhanced interactivity and feedback not previously utilized in a conference setting. 


\section{References}

1. Rosenberg M, ed: E-learning: strategies for delivering knowledge in the digital age. New York: McGraw-Hill, 2001

2. Ruiz JG, Mintzer MJ, Leipzig RM: The impact of E-learning in medical education. Acad Med. Mar 81(3):207-212, 2006

3. Tidmarsh PJ, Cummings J, Hersh WR, Freidman CP: Distributed medical informatics education using internet2. Proc AMIA Symp 2002:787-791

4. Davidson HC, Wiggins RH: Radiology teaching presentation tools. Semin Ultrasound CT MR 24(6):420-427, 2003
5. Wiggins RH: Personal digital assistants. J Digit Imaging 17(1):517, 2004

6. Rowell MR, Johnson PT, Fishman EK: Radiology education in 2005: world wide web practice patterns, perceptions, and preferences of radiologists. Radiographics 27(2):563-571, 2007

7. Accreditation Council for Continuing Medical Education www. accme.org. Accessed 20 May 2011

8. Weiner SJ, Jackson JL, Garten S: Measuring continuing medical education outcomes: a pilot study of effect size of three CME interventions at an SGIM annual meeting. J Gen Intern Med 24 (5):626-629, 2009 